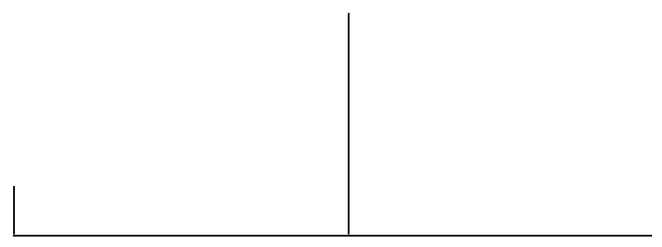

Rev. Latinoam. Psicopat. Fund., São Paulo, v. 15, n. 1, p. 108-112, março 2012

\title{
Jean Colombier e François Doublet: o nascimento da psiquiatria
}

\author{
Manoel Tosta Berlinck
}

Escrito por Jean Colombier e François Doublet, "Instruções sobre como controlar os insanos e de trabalhar para sua cura nos Asilos que lhes são destinados", foi o primeiro documento de caráter reformador que se ocupou da situação dos doentes mentais, indicando os critérios que a psiquiatria do século XIX deveria seguir.

Teve sua importância histórica reconhecida, apesar de sua breve vigência, tendo, a seguir, o seu conteúdo sido assimilado pelo Comitê de Mendicidade da Assembleia Constituinte.

Palavras-chave: Saúde mental, história da psiquiatria, tratamento 
Em 1785, Jean Colombier e François Doublet escreveram Instruções para governar os insensatos, por solicitação do governo francês, antes de terem sido nomeados pelo rei Luis XVI, inspetor geral e inspetor adjunto dos Hospitais civis e das prisões do Antigo Regime.

Este informe, que foi amplamente difundido por todo o reino, conserva o grande interesse de ser o primeiro documento de caráter reformador que, imediatamente antes da Revolução, se ocupou da situação dos doentes mentais e indicou os critérios que a psiquiatria do século XIX deveria seguir.

Jean Colombier (1736-1789), cirurgião do exército, encarregou-se da redação da primeira parte, "que concerne sobre a maneira de internar, guardar e dirigir os insensatos", enquanto François Doublet (1751-1795), especialista em doenças venéreas, professor da Faculdade de Medicina de Paris e membro da Sociedade Real de Medicina, se ocupou da segunda, "O tratamento dos insensatos". Antes de redigir as Instruções, viajaram pela França visitando hospitais e foram para a Inglaterra com o intuito de conhecer as inovações mais recentes. Entretanto, depararam-se com abusos e preconceitos com que eram tratados todos os loucos e criticaram os modernos por haverem colocado em um mesmo lugar todas as espécies de loucura, visando ficar livres dela em vez de cuidarem dos doentes. É importante observar que, desde a ordem real de 1767 que regulava a mendicidade, os hospitais se converteram numa instalação confusa saturada de velhos e indigentes.

Guiados por um critério social novo, os redatores do informe observaram que, assim como os ricos podiam cuidar de seus parentes, os pobres eram incapazes de fazê-lo, argumentando que a sociedade deveria proteger os seres mais fracos e desgraçados. Junto com esse tom filantrópico, as Instruções propõem um critério assistencial completamente novo. De fato, o documento de Colombier e Doublet é o primeiro projeto oficial de medicalização da loucura na França. Desde então, os poderes do Estado foram admitindo pouco a pouco a necessária responsabilidade dos alienistas, enquanto ao alienado foi reconhecida sua condição de doente. O sistema havia deslocado finalmente seu centro de gravidade do triângulo formado pelo juiz, a família e a administração, e o médico passou a ser figura central nos cuidados com a loucura.

Na primeira parte das Instruções, Colombier apresenta as condições materiais que devem apresentar os estabelecimentos para evitar que os males da internação dos alienados cresçam e que o fim das doenças coincidam com o

Rev. Latinoam. Psicopat. Fund., São Paulo, v. 15, n. 1, p. 108-112, março 2012 
fim de seus dias. Além desses aspectos, o autor trata da necessária distribuição dos doentes e das bases do que haveria de constituir o futuro tratamento moral da loucura. Os fundamentos terapêuticos da instituição obtêm também cuidadosa atenção, antes de Esquirol sustentar que "uma casa de alienados é um instrumento de tratamento; nas mãos de um hábil médico, é o agente mais potente contra as doenças mentais". A estranha combinação de alienista, higienista e filantropo que iria definir os primeiros psiquiatras do século XIX, encontra seu germe nesse texto.

Doublet, por sua vez, animado pela necessidade de colocar ordem na massa indistinta da loucura, propõe diferenciar quatro tipos básicos de alienação: frenitis, mania, melancolia e imbecilidade. Em outras palavras, alienação com febre, alienação total, alienação parcial - limitada a um só objeto, o "ponto melancólico" - e alienação por deficiência intelectual. Classificação que, por sua origem e concisão, aspirava a certa oficialidade como guia para os futuros reformadores de hospitais. No que se refere ao tratamento, Doublet, em sua breve proposta e querendo evitar qualquer pessimismo terapêutico, recomenda todos os recursos médicos tradicionais: sangrias, purgantes, escarificações, banhos, duchas, isolamento etc.

A importância histórica das Instruções foi reconhecida, apesar de sua vida breve. A Revolução deteve o projeto ainda que, mais tarde, o novo Comitê de Mendicidade da Assembleia Constituinte tenha assimilado seu conteúdo.

Por outro lado, o que poderia ser entendido como uma inovação progressista, a medicalização do louco se transformou numa hospitalização totalitária, denunciada cuidadosamente por Foucault.

Entretanto, não se pode ignorar a importância das Instruções, onde estão formulados os fundamentos da psiquiatria francesa, antes mesmo da Revolução.

\section{Resumos}

(Jean Colombier and François Doublet: the Birth of Psychiatry)

Jean Colombier and François Doublet were the authors of Instructions on How to Control the Insane and Work toward their Cure in Asylums to Which They Are Sent. This book was the first document of a reformist character about the situation of mental patients, and indicated criteria that 19th-century psychiatry should follow. Even though it was in print for only a short time, it became a highly respected work, as its contents were subsequently assimilated by the Committee on Begging at the First Constitutional Assembly, convened to write the Brazilian Constitution.

Key words: Mental health, history of psychiatry, treatment 


\section{CLÁSSICOS DA PSICOPATOLOGIA}

(Jean Colombier et François Doublet: la naissance de la psychiatrie)

Écrit par Jean Colombier et François Doublet, les "Instruction sur la manière de gouverner les insensés et de travailler à leur guérison dans les asyles qui leur sont destinés" est le premier document de caractère réformateur qui examine la situation des malades mentaux et qui défini les critères que la psychiatrie du XIX siècle devrait suivre. Son importance historique est reconnue, bien qu'elle ne soit restée en vigueur que peu de temps, puisque son contenu a été assimilé par le Comité de Mendicité de l'Assemblée Constituante.

Mots clés: santé mentale, histoire de la psychiatrie, traitement

(Jean Colombier y François Doublet: el nacimiento de la psiquiatria)

Escrito por Jean Colombier y François Doublet, "Instrucciones sobre como controlar a los insanos y de trabajar para su curación en los Asilos a que son destinados", fue el primer documento de carácter reformador que se ocupó de la situación de los enfermos mentales, indicando los criterios que la psiquiatria del siglo XIX deberia seguir.

Tuvo su importancia histórica reconocida a pesar de su breve vigencia, mas su contenido fue asimilado por la Comisión de la Medicación en la Asemblea Constituyente.

Palabras clave: Salud mental, historia de la psiquiatria, tratamiento

(Jean Colombier und François Doublet: Die Geburt der Psychiatrie)

"Anleitung zum Umgang mit Geistesgestörten und die Arbeit zu ihrer Heilung in den für sie bestimmten Anstalten”, von Jean Colombier e François Doublet, war das erste wirklich reformatorische Dokument, das sich mit der Situation der Geisteskranken beschäftigte, indem es Kriterien zusammenstellte, die der Psychiatrie des 19. Jahrhunderts als Leitfaden dienen sollten.

Trotz seinem kurzen Bestehen, wurde seine historische Bedeutung anerkannt und sein Inhalt, anschließend vom Comitê de Mendicidade da Assembleia Constituinte (Bettler-Komitee der Verfassungsgebenden Versammlung) aufgenommen.

Schlüsselwörter: Geistige Gesundheit, Geschichte der Psychiatrie, Behandlung

Citação/Citation: Berlinck, M.T. Jean Colombier e François Doublet. Revista Latinoamericana de Psicopatologia Fundamental, São Paulo, v. 15, n. 1, p. 108-112, mar. 2012.

Editor do artigo/Editor: Prof. Dr. Mario Eduardo Costa Pereira 


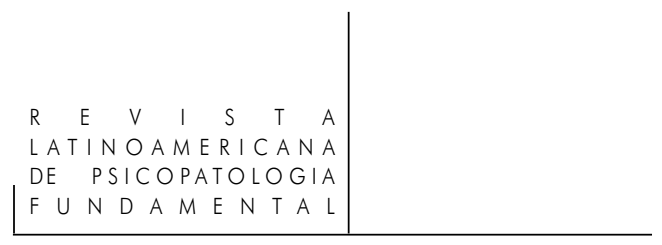

Recebido/Received: 1.2.2012 / 2.1.2012 Aceito/Accepted: 5.2.2012 / 2.5.2012

Copyright: () 2009 Associação Universitária de Pesquisa em Psicopatologia Fundamental/ University Association for Research in Fundamental Psychopathology. Este é um artigo de livre acesso, que permite uso irrestrito, distribuição e reprodução em qualquer meio, desde que o autor e a fonte sejam citados/This is an open-access article, which permits unrestricted use, distribution, and reproduction in any medium, provided the original author and source are credited.

Financiamento/Funding: $\mathrm{O}$ autores declara não ter sido financiado ou apoiado/The author has no support or funding to report.

Conflito de interesses/Conflict of interest: $O$ autor declara que não há conflito de interesses/The author declares that has no conflict of interest.

\section{Manoel Tosta Berlinck}

Sociólogo; Psicanalista; Ph.D. (Cornell University, Ithaca, N.Y., USA); Professor Titular da Universidade Estadual de Campinas - Unicamp (1972-1992) (Campinas, SP, Br.); Professor do Programa de Estudos Pós-Graduados em Psicologia Clínica da Pontifícia Universidade Católica de São Paulo - PUC-SP (São Paulo, SP, Br.), onde dirige o Laboratório de Psicopatologia Fundamental; Presidente da Associação Universitária de Pesquisa em Psicopatologia Fundamental - AUPPF (2002 - 2010) (São Paulo, SP, Br.); Diretor da Editora Escuta (1986-2009); Diretor da Livraria Pulsional (1986-2009); Consultor editorial; Editor responsável da Revista Latinoamericana de Psicopatologia Fundamental; Membro da World Association of Medical Editors - WAME (Associação Mundial de Editores Médicos); Autor de Psicopatologia Fundamental (2000) e de Erotomania com German E. Berrios (2009), entre outros livros e numerosos artigos.

Rua Tupi, 397/103

01233-001 São Paulo, SP

Fonefax: 55113825.8573

e-mail: mtberlin@uol.com.br 